\title{
Vom Handwerk des Chemiehistorikers
}

Der Geschichte der Naturwissenschaften wird gegenwärtig wachsende Aufmerksamkeit geschenkt. Nicht nur die Naturwissenschaftler selbst, sondern auch Wissenschaftsdidaktiker, Lehrer, Wissenschaftsjournalisten und Studenten der unterschiedlichsten naturwissenschaftlichen Fächer verfolgen die Ergebnisse der wissenschaftshistorischen Forschung oder beginnen auch, eigene Fragen an die Geschichte ihrer Disziplin zu richten. Unter Allgemeinhistorikern ist es inzwischen unbestritten, daß die Geschichte der Wissenschaft einen wesentlichen Beitrag zur Menschheitsgeschichte liefert, greifen doch Naturwissenschaft und Technik in immer stärkerem Maße in die historische Entwicklung ein. Die Motive für das neuerwachte Interesse an der Geschichte der Naturwissenschaften sind freilich unterschiedlich. Konnte noch das ausgehende 19. Jahrhundert in ungetrübtem Stolz auf die Errungenschaften der Vergangenheit blicken und sich selbst dem Ziel einer lückenlosen Naturerklärung und Naturbeherrschung nahe wähnen, so überwiegt heute der Eindruck, daß ein Wendepunkt im exponentiellen Wachstum erreicht und der Fortschrittsgedanke selbst an eine Grenze gekommen ist, wird doch die gegenwärtige Krise weitgehend als eine Krise des naturwissenschaftlich-technischen Denkens empfunden. Sie wird wohl nur dann zu überwinden sein, wenn es gelingt, die Kluft zwischen den "beiden Kulturen“, der geisteswissenschaftlich-literarischen Intelligenz auf der einen und der naturwissenschaftlich-technischen Intelligenz auf der anderen Seite, die sich seit Beginn des 19. Jahrhunderts in bedenklicher Weise vertieft hat, wieder zu schließen und damit der verhängnisvollen Auseinanderentwicklung von emotionalethischer Wertorientierung und rationalpragmatischer Sachorientierung Einhalt zu gebieten. Wo aber der eigene Standort nicht mehr selbstverständlich ist, sondern von Grund auf neu bestimmt werden muß, dort gewinnt die historische Dimension an Aktualität. Denn eine jede Positionsbestimmung in der Gegenwart hat sich nicht nur vor der eigenen Zukunftserwartung, sondern auch vor der eigenen Vergangenheit zu rechtfertigen.

Es wäre nun freilich verfehlt, vom Historiker Patentrezepte zu erwarten. Die Geschichte hält kein derartiges fabula docet bereit. Der Historiker gibt ebensowenig den besseren Politiker $a b$ wie der Kunsthistoriker den kreativeren Maler oder der Chemiehistoriker den besseren Chemiker. Wir können die $\mathrm{Zu}$ versicht William Whewells nicht mehr teilen.

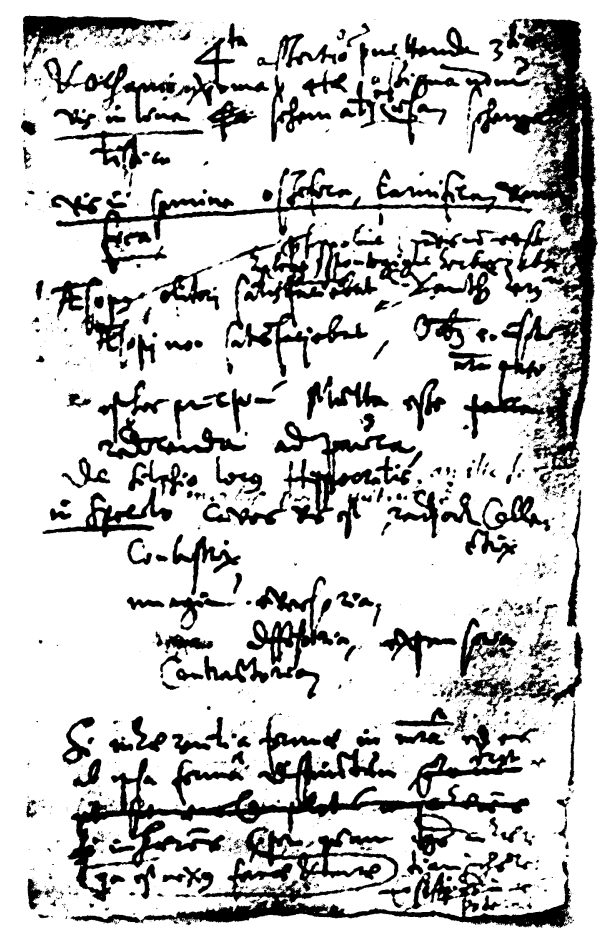

Die Arbeit mit handschriftlichen Quellen erschließt einen besonders unmittelbaren Zugang zur Vorstellungswelt einer vergangenen Epoche. Hier ein nach 1630 angelegtes Notizblatt von Joachim Jungius zur Methodenlehre der Naturwissenschaft [Staats- und Universitätsbibliothek Hamburg, Nachlaß Jungius, Sign. Pe. 51 a, fol. 135].

der seine "History of the Inductive Sciences", die erste allgemeine Geschichte der $\mathrm{Na}$ turwissenschaft, 1837 in der Hoffnung herausgegeben hatte, der historisch gebildete Naturwissenschaftler werde auch der bessere und erfolgreichere Forscher sein, weil er im Studium der Vergangenheit Lösungswege kennen und Irrwege vermeiden gelernt habe.

Zu Whewells Zeit freilich wurde die Geschichte der Naturwissenschaften noch ausschließlich von Naturwissenschaftlern für Naturwissenschaftler geschrieben. Die Reihe der chemiehistorischen Darstellungen aus der Feder großer Chemiker reicht von Johann Friedrich Gmelin (1797-99) über Hermann Kopp (1843-47) bis zu Paul Walden (1947) und James Riddick Partington (1961-70). Im Laufe der Zeit aber sind wissenschaftshistorische Forschung und die sich immer weiter differenzierenden Naturwissenschaften jeweils eigene Wege gegangen. Heute stehen sie sich als zwei autonome Fachrichtungen gegenüber, die über spezifische Arbeitsgebie- te und Forschungstraditionen, eigene Fachzeitschriften und Organisationen verfügen. Für den Dialog ist diese Ausgangsposition im Grunde nicht ungünstig, vorausgesetzt, beir de Seiten bemühen sich um das Gespräch über die Fachgrenzen hinweg. Interdiszipli narität setzt stets die Disziplinarität der Dialogpartner voraus.

Was aber darf der Naturwissenschaftler, der Chemiker, von diesem Dialog erwarten: worin liegt denn nun der Wert der Geschich te für die Gegenwart, wenn denn die Hoff nung, in ihr Patentlösungen vorzufinden. vergeblich ist? Die Antwort muß und dar subjektiv ausfallen, wie ja auch die Erwar tungen an die Geschichte vom jeweiligen Standpunkt abhängen. Ich will deshalb auch nur einen mir besonders wichtigen Aspekt herausstellen.

Die naturwissenschaftlich-technische Zivilisation, in der wir leben, wird als ein Syster höchst komplexer Zusammenhänge erfahren. das sich der persönlichen Einflußnahme weitgehend entzieht. Wir lernen, uns in einer Welt objektiver Sachzwänge und vorgefur dener Gegebenheiten zurechtzufinden, in der für menschliche Subjektivität immer weniges Platz zu bleiben scheint. Wir eignen uns dir Naturwissenschaft, die wir studieren und ausüben, als ein wohlbegründetes System festliegender Ergebnisse und Sätze an. Aw dieser Einsicht in die Notwendigkeit und $B$ dingtheit der Verhältnisse stellt sich die Ge schichte als Ort der Freiheit dar, von dem aw die Gegenwart neu in den Blick gerät. Dent was in ihr als geworden erfahren wird, er weist sich historisch als werdend; was in ibt statisch und endgültig erscheint, zeigt sich historisch als dynamisch und stets nur vor läufig. Anstelle des Resultates zeigt die Ge schichte den Prozeß, anstelle des Zieles des Weg. Vom Bedingtsein und der normative Kraft des Faktischen verweist sie auf die Freis heitsgrade von Veränderung und das Spektrum des Möglichen. Darüber hinaus lenkt sie, und dies ist nicht ihr geringstes Ver dienst, den Blick immer wieder auf das hu manum, auf den Menschen, als das empfin dende, denkende, handelnde, aber auch ir rende Subjekt aller Geschichte. Es ist deshalb keine Flucht vor der Gegenwart und ihred Aufgaben, wenn sich der Historiker der Ver gangenheit zuwendet, sondern er tut dies, um die für das Verständnis der Gegenwart notwendige, kritische Distanz zu gewinnen und um die damit erworbene Freiheit auch der Zukunft mit auf den Weg zu geben. 
Im folgenden soll der Versuch unternommen werden, Gegenstand und Methode der Chemiegeschichtsschreibung näher zu bestimmen. Eine derartige Einführung kann selbstverständlich die Erfahrung nicht ersetzen, die man erst im Umgang mit den Quellen, der Literatur und den Hilfsmitteln der Wissenschaftsgeschichte erwirbt. Wie der Chemiker sein Handwerkszeug nur bei der Arbeit im Labor erwerben kann, so läßt sich auch das Handwerk des Chemiehistorikers nicht aus einer solchen Einführung erlernen. Der Versuch, Historiker oder Philologen in ähnlich knapper Form das chemische Experimentie-

Chemisches Labor mit Ófen und Destillationsvorrichtungen. Stammbuchblatt von Philipp Georg Dietrich aus Leipzig für den Pharmaziestudenten Wolfrad Wilhelm Mair, Dresden 1638 Mrz 20 (Germanisches Nationalmuseum Nürnberg, Sign. HB 13053a). Bildliche Darstellungen erfordern eine besonders behutsame Interpretation und dürfen nicht mit photographischen Wiedergaben von Wirklichkeit verwechselt werden. [Zur Quellenkritik von Abbildungen vgl. C. R. Hill: The Iconography of the Laboratory. Ambix 22, 102 (1975)] ren zu lehren, dürfte sich schon aus Sicherheitsgründen verbieten. Im Bereich der Historiographie liegen die Gefahren freilich nicht so offen auf der Hand. Das wichtigste Ziel der vorliegenden Ausführungen ist deshalb, Verständnis für die Eigenart der Chemiegeschichtsschreibung zu wecken, um damit den Dialog zwischen Chemie und Chemiegeschichte $\mathrm{zu}$ intensivieren.

\section{Der Gegenstand der Chemiegeschichte}

Die Geschichte der Chemie ist, wie ihr Name sagt, eine historische Disziplin und keine naturwissenschaftliche. Ihr Gegenstand freilich ist die Art und Weise, in der Menschen zu früheren Zeiten über stoffliche Vorgänge und Erscheinungen in der Natur nachgedacht, ihre Erfahrungen und Gedanken formuliert und schließlich in praktisches Tun umgesetzt haben. Die Versuche, das Wesen von Stoff und Eigenschaft zu begreifen, sind so alt wie menschliches Nachdenken über die Natur insgesamt. Nicht die Natur chemischer Vorgänge ist also Gegenstand der Chemiegeschichte, sondern der Dialog des Menschen mit der Natur in seiner historisch sich wandelnden Gestalt. Um ihn zu verstehen, bedarf es zunächst der Methoden des Philolo- gen und des Historikers, nicht der des $\mathrm{Na}$ turwissenschaftlers. Die Sache aber, um die es ging, erfordert darüber hinaus das Fachwissen des Chemikers. Hierin liegt die erste Schwierigkeit unseres Vorhabens.

Eine zweite kommt hinzu. Denn es kann in der Geschichtsschreibung ja nicht darum gehen, die Vergangenheit vom gleichfalls vergänglichen Standpunkt der Gegenwart aus zu beurteilen, über richtig und falsch zu richten oder gar Lob und Tadel auszuteilen. Vielmehr geht es darum, der Vergangenheit zu ihrem eigenen Recht zu verhelfen, indem wir sie von ihren eigenen Voraussetzungen, Bedingungen und Möglichkeiten her verstehen. Gerade dies macht den Reiz, aber auch die Schwierigkeit der Historiographie aus. Denn so lange wir "nostrizentrisch" die Vergangenheit ausschließlich von unserer Warte her betrachten und sie mit unseren Maßstäben messen, werden wir in ihr, wenn auch im fernen Spiegel, nur wiederum uns selbst erblicken. Geschichtsschreibung sollte, so weit dies überhaupt zu leisten ist, ,idiozentrisch“ vorgehen, sich in die Vergangenheit hineinbegeben, sich ihre Maßstäbe zu eigen machen und sie mit den ihr angemessenen Kriterien beschreiben, selbst auf die Gefahr hin, daß

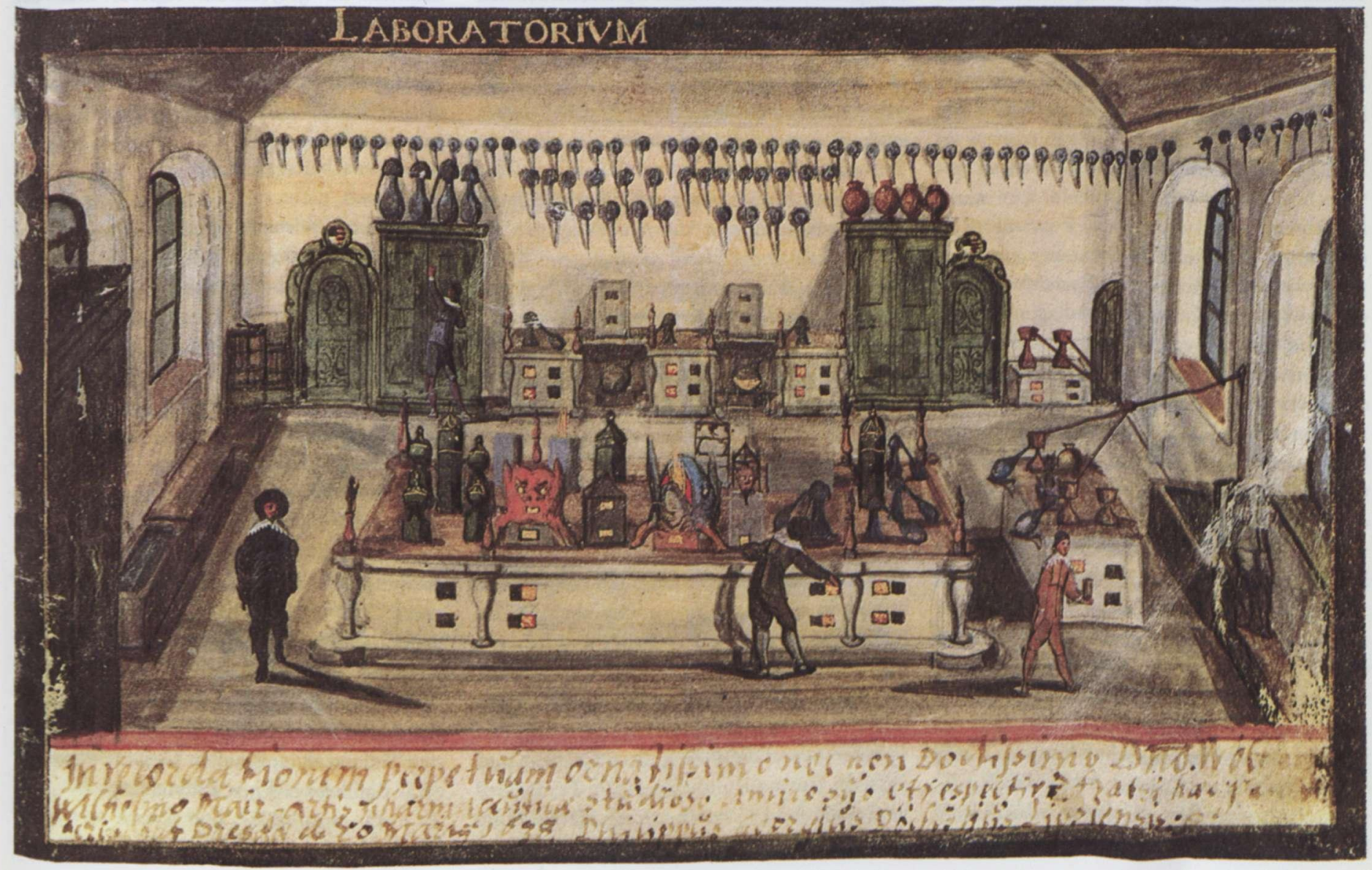


dort, wie in Lewis Carrols Geschichte von Alice "Through the Looking-Glass", die uns vertrauten Denkgewohnheiten keine Geltung mehr besitzen.

Das Wissen einer vergangenen Epoche war ja nicht bloß ein quantitativ geringeres Wissen, sondern es war qualitativ von dem unseren verschieden. Je weiter wir uns von der Gegenwart entfernen, um so deutlicher treten die Unterschiede hervor. Man vergleiche dazu nur einmal ein Lehrbuch der Organischen Chemie aus dem ersten Drittel des 19. Jahrhunderts mit einem entsprechenden Werk unserer Tage und wird Mühe haben, in beiden das gleiche Fachgebiet zu erkennen. Wie sehr muß dann erst die chemische Kosmologie der Paracelsisten oder gar die Chemie des Aristoteles von der unseren verschieden gewesen sein. Der Fortgang der Wissenschaft besteht eben nicht bloß in der Kumulation gesicherter Fakten und der Ablösung untauglicher Theorien durch bessere. Er bringt vielmehr eine ständige Neuordnung des Gesamtsystems einer Wissenschaft mit sich, so daß man oft nicht einmal davon sprechen kann, daß ein isoliertes Faktum im gänzlich veränderten Beziehungsgefüge gleich geblieben sei. Andere Zeiten stellten andere Fragen und erwarteten andere Antworten. Man machte es sich zu einfach, wollte man glauben, im Laufe der Wissenschaftsentwicklung sei die Zahl der offenen Fragen geringer und die der gesicherten Antworten größer geworden.

Den Historiker stellt dieser Sachverhalt natürlich vor erhebliche Probleme. Um nämlich einen Chemiker, sagen wir aus der Zeit der Romantik, recht zu verstehen, muß er nicht nur dessen Beitrag zur Fortentwicklung der wissenschaftlichen Chemie einschätzen können, sondern er müßte im Grunde auch die Chemie der Zeit um 1800 in gleicher Weise beherrschen wie die Zeitgenossen, um deren Voraussetzungen, Fragen und Möglichkeiten richtig zu bewerten. Dies aber bedeutete, strenggenommen, nicht weniger, als das eigene chemische Wissen, das leidige Besserwissen derer, die vorerst das letzte Wort behalten, in einem rückwärtigen Paradigmenwechsel für einen Moment zu vergessen. Doch damit nicht genug. Als Historiker haben wir es ja nicht mit immateriellen Ideen zu tun, sondern naturwissenschaftliche Vorstellungen und Resultate treten uns stets in Gestalt wirklicher Menschen entgegen und wollen deshalb im Kontext der gesellschaftlichen, institutionellen, geistigen und kulturellen Bedingungen gesehen werden, unter de- nen diese so und nicht anders gedacht und gehandelt haben.

\section{Zur Methode der Chemiegeschichte}

Der Begriff "Methode" bezeichnet in der Geschichtswissenschaft zweierlei. Zum einen belegt man damit das systematische Vorgehen bei der Ermittlung der Quellen (Heuristik), ihrer kritischen Prüfung und ihrer Interpretation. Zum anderen bezeichnet Methode die Eigenart historischen Erkennens überhaupt. Dieses unterscheidet sich nämlich fundamental von dem der Naturwissenschaften: Während diese aus der Erscheinungsvielfalt der Natur experimentierend und quantifizierend allgemeine Gesetzmäßigkeiten $a b$ zuleiten suchen, geht es in der Geschichtswissenschaft zunächst darum, ein Ereignis oder einen Lebenslauf in seiner Einmaligkeit und Unwiederholbarkeit zu erfassen und zu verstehen. Denn anders als in Physik und Chemie läßt sich das historische Geschehen nicht als eine notwendige Folge zugrundeliegender Naturgesetze begreifen, auch wenn man sich neuerdings bemüht, allgemeinere Strukturen im geschichtlichen Prozeß deutlicher herauszuarbeiten.

An einem zentralen Punkt unterscheidet sich die Geschichte der Chemie als historische Disziplin besonders scharf von einer Naturwissenschaft wie der Chemie. Es ist dies die nur scheinbar triviale Tatsache, daß Vergangenheit vergangen und damit der direkten Beobachtung ein für allemal entzogen ist. Chemiker, Physiker oder Biologen können den Gegenstand ihrer Untersuchung im klassischen Fall in die Hand nehmen, mit Meßinstrumenten untersuchen, den unterschiedlichsten experimentellen Prüfungen unterwerfen und diese so oft wiederholen, wie es erforderlich scheint. Dem Historiker ist all dies verwehrt. Die Personen, mit denen er es zu tun hat, sind tot, die Ereignisse, die er beschreibt, unwiederbringlich vergangen.

Wer sich deshalb als Chemiehistoriker mit Leben und Werk eines Chemikers aus dem 19. Jahrhundert befaßt, wird den Gegenstand seiner Studien niemals zu Gesicht bekommen. Was er von ihm in Erfahrung bringen kann, verdankt er ausschließlich Zeugnissen der Vergangenheit, die die Zeiten mehr oder minder zufällig überdauert haben. Der $\mathrm{Hi}$ storiker nennt diese Zeugnisse Quellen und versteht darunter alle diejenigen Texte, Gegenstände oder Tatsachen, mit deren Hilfe sich Kenntnis von der Vergangenheit gewin- nen läßt. Ganz unterschiedliche Quelle können von der Entdeckung einer neuel Verbindung Zeugnis geben: das Labortage buch des Entdeckers, seine briefliche Mitter lung an einen Kollegen, die Publikation in er ner Fachzeitschrift, ein Bericht in der Tages presse, ein Interview anläßlich einer Preisver leihung, die Gründung einer Firma, die dix neue Substanz produzierte, aber auch die Le benserinnerung eines Schülers, der seinerzed dabei war, ein Inventarverzeichnis des Labo ratoriums, die Apparatur, mit der die Syn these gelang, oder gar eine erhaltene Sub stanzprobe. Die wenigsten dieser Zeugnisst werden eigens deshalb angefertigt und aufbe wahrt worden sein, um uns, die Nachwelt. über jene Entdeckung und die Umstände, dir zu ihr führten, zu unterrichten. In der Regd werden wir es vielmehr mit unmittelbare Uberresten des historischen Ereignisses 24 tun haben, die im Kontext ihrer Zeit eine ganz bestimmte Funktion besaßen und dan eher durch Zufall auf uns gekommen sind.

Sie alle geben natürlich irgendwie Kunde vo dem Ereignis, für das wir uns als Historiker interessieren. Quellenkunde im strengeres Sinne bedeutet nun, dieses unbefriedigende "irgendwie" in methodisch gesicherte Er kenntnis zu überführen. Hierfür muß der Wissenschaftshistoriker beim Allgemeinhistoriker in die Lehre gehen, auch wenn des' sen Typologie der Quellen den Verhältnisse? in der Wissenschaftsgeschichte nicht immer ganz adäquat ist. Eine Quellenkunde der Naturwissenschaftsgeschichte aber gibt es bislang noch nicht. Es ist nämlich durchaus nicht so, als seien die Quellen an sich schon Geschichte oder gar das Ziel der historischen Erkenntnis. Vielmehr spiegeln Quellen als unmittelbarer Niederschlag geschichtliched Handelns jeweils nur bestimmte Aspekte der historischen Wirklichkeit. Quellen sind deshalb nicht nur interpretierbar, sondern ihrem Wesen nach interpretationsbedürftig. In ihren Entstehungszusammenhang eingebunden, spiegeln sie Interessen, Zielsetzungen, aber auch Fehleinschätzungen ihrer Schreiber oder Hersteller. So berichten sie uns die Entdeckungsgeschichte eines neuen Elements unter Umständen ganz verschieden, je nachdem ob uns ein Labortagebuch, eine Fachpublikation, eine Apparatur, ein Brief des Entdeckers an seine Frau oder ein Nachruf aus der Feder seines Schülers vorliegen. Im be sten Fall erzählen Quellen Geschichten, und zwar die ihrer eigenen Entstehung.

Geschichte im eigentlichen Sinne des Wortes 


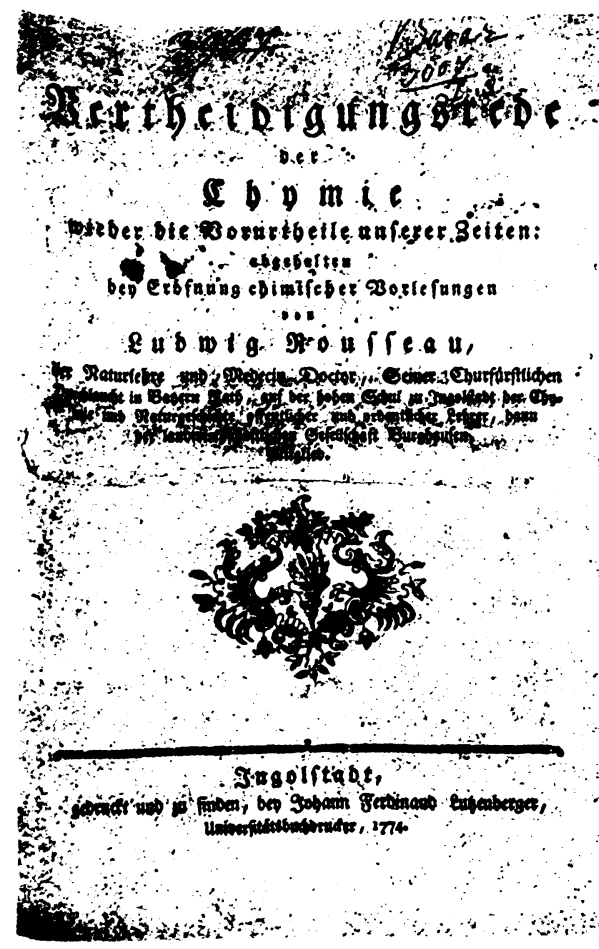

Selbstdarstellungen der Chemie, wie sie Antrittsvorlesungen, Vorworte und propädeutische Schriften überliefern, sind auBerordentlich wichtige Zeugnisse, müssen aber kritisch auf ihre Intention und Tendenz hin befragt werden. [Vgl. auch Christoph Meinel: De praestantia et utilitate Chemiae. Selbstdarstellung einer jungen Disziplin im Spiegel ihres programmatischen Schrifttums. Sudhoffs Archiv 65, 366 (1981)]

wird daraus erst dann, wenn der Historiker diese Einzelaspekte unter einer bestimmten Fragestellung in einen inneren Zusammenhang bringt und zu einem Gesamtbild vereinigt. Geschichte und ihr Gegenstand entstehen also erst als Resultat einer bewußten $\mathrm{Zu}$ sammenschau. Mit objektiven Tatsachen im Sinne naturwissenschaftlicher Erkenntnis hat es die Geschichtswissenschaft niemals zu tun. Einer der großen Geschichtstheoretiker des vergangenen Jahrhunderts, Johann Gustav Droysen, hat einmal gesagt: „Es heißt die Natur der Dinge, mit denen unsere Wissenschaft beschäftigt ist, verkennen, wenn man meint, es da mit objektiven Tatsachen zu tun zu haben. Die objektiven Tatsachen liegen in ihrer Realität unserer Forschung gar nicht vor. ... Was geschieht, wird erst durch die Auffassung als zusammenhängender Vorgang, als ein Komplex von Ursache und Wirkung, von Zweck und Ausführung, kurz als eine Tatsache begriffen und vereinigt, und dieselben Einzelheiten können von anderen anders aufgefaßt, sie können von anderen mit anderen Ursachen oder Wirkungen oder Zwecken kombiniert werden" (Enzyklopädie und Methodologie der Geschichte, \33). Diesem Urteil ist schon deshalb beizupflichten, weil die Quellen niemals die historische Wirklichkeit in ihrer Gesamtheit widerspiegeln. Sie sind punktuelle Zeugnisse, die der Historiker interpretieren und zueinander in Beziehung setzen muß, will er sich mit ihrer Hilfe der Vergangenheit vergewissern.

Aufgabe der Quellenkritik ist es nun, die Verläßlichkeit und Bedeutung einer Quelle festzustellen. Dies muß sowohl in formaler als auch in inhaltlicher Hinsicht geschehen. Bei der formalen Kritik geht es zunächst um die Identifikation und Echtheitsprüfung, die gerade bei gegenständlichen Quellen oft Schwierigkeiten bereitet, da diese weniger beredt sind als die schriftlichen Zeugnisse. In der Textkritik hingegen ist vorab häufig philologische Arbeit zu leisten, wenn es etwa darum geht, aus einer möglicherweise fehlerhaften Uberlieferung einen verläßlichen Text zu erstellen. Bei geschäftlichen Schriftstükken unterrichtet die Aktenkunde über die formalen Eigenheiten, aus denen sich der unterschiedliche Quellenwert von Konzepten, Abschriften, Ausfertigungen und dem Empfänger präsentierten Stücken ergibt. Aber auch bei gedruckten Quellen werden wir uns immer textkritisch fragen müssen, ob die uns vorliegende Fassung wirklich der Schreibintention des Autors gerecht wird. Bis ins 19. Jahrhundert hinein war es nämlich die Ausnahme, daß ein Autor Korrekturfahnen seines Werkes erhielt; Ubersetzungen fachwissenschaftlicher Texte bereiteten ungeahnte Schwierigkeiten, und es gab wenig Schutz gegen unautorisierte Nachdrucke. Jöns Jacob Berzelius, der große schwedische Chemiker, hat selbst bitter genug erfahren müssen, wie hinderlich sich schlechte Ubersetzungen und fehlerhafte Raubdrucke auf die Verbreitung seiner Ansichten auswirkten. Schließlich muß der Historiker, der etwa einen Briefwechsel herausgeben will, wissen, welche Grundsätze der Textgestaltung dafür üblich und sinnvoll sind.

Während die Textkritik vornehmlich auf formale Gesichtspunkte zielt, geht es in der Sachkritik um die Frage, wie sich der Wortlaut der Quelle zum wirklichen historischen Ereignis verhält. Eine allgemeine Richtschnur zu ihrer Beantwortung läßt sich hier natürlich nicht angeben, weil die Argumente der Sachkritik inhaltlicher Natur sind und deshalb nur von Fall zu Fall entwickelt werden können. Zwei Beispiele sollen das Problem beleuchten:

Im Revolutionsjahr 1848 schickte Eduard Platner, Professor für Römisches Recht an der Universität Marburg, der kurhessischen Ständeversammlung ein Gutachten über den Zustand seiner Hochschule und die Notwendigkeit von Reformen. Besonders hart ging er dabei mit der Medizinischen Fakultät ins Gericht:

„Professor Fick, der Anatom, ist ein junger Mann von einigem Talent, überläßt sich aber, seitdem er ordentlicher Professor geworden ist, einer Trägheit, die einen sehr geringen Grad von geistiger Elasticität verräth, und ist daher sowohl als Lehrer wie als Gelehrter durchaus unbedeutend geblieben. ... Professor Hüter, der Geburtshelfer, ist ein tüchtiger Practiker, allein der unfähigste Theoretiker, den wir in unserem Leben zu hören Gelegenheit hatten; seine Vorlesungen zu besuchen ist eine starke Zumuthung, deshalb begreiflich, daß die Studenten aus denselben systematisch weggeblieben sind. ... Professor Ullmann ist eine vollkommene Null. Schon seit längerer Zeit ohne Zuhörer und von dem Directorium der chirurgischen Klinik entbunden, hat man ihm doch noch immer Sitz und Stimme in der Facultät gelassen, obschon er so schwach und stumpf ist, daß er oft heute nicht mehr weiß, was er gestern gesagt hat."

So erfrischend eine derart deutliche Sprache sein mag, so unverzeihlich wäre es, wollte man die Aussagen ungeprüft für bare Münze nehmen. Selbstverständlich mag Platners hartes Urteil zutreffen. Um dies aber wirklich behaupten zu können, müßten wir uns zuvor Rechenschaft darüber gegeben haben, wer der Autor war, woher er seine Kenntnisse bezog, und wie er als Jurist dazu kam, derart über seine Kollegen von der Medizinischen Fakultät herzuziehen. Was mag er mit seiner Eingabe bezweckt haben, und was war in dieser Hinsicht von der Kasseler Ständeversammlung zu hoffen? Hat sein Vorstoß am Ende Gegendarstellungen provoziert, die uns helfen könnten, sein Urteil ins rechte Verhältnis zu rücken?

Eine Quelle allein ist eben nie die geschichtliche Wirklichkeit selbst, sondern reflektiert Wirklichkeit durch das Medium des jeweiligen Schreibers. Erst wenn wir die Umstände 
ihrer Entstehung genau kennen, wenn wir wissen, weshalb sie die Dinge so und nicht anders darstellt, werden wir ihren historischen Gehalt erfassen können. Ein zweites Beispiel mag die Bedeutung quellenkritischer Uberlegungen noch einmal unterstreichen.

Welcher Chemiker hat die Geschichte von der Entdeckung der Benzolformel durch Kekulé nicht oft genug gehört, jene Geschichte von der durchwachten Nacht am Genter Kaminfeuer, in dessen Funkenspiel dem schlaftrunkenen Betrachter die lang gesuchte Lösung aufschien: „Eine der Schlangen erfaßte den eigenen Schwanz und höhnisch wirbelte das Gebilde vor meinen Augen. Wie durch einen Blitzstrahl erwachte ich; auch diesmal verbrachte ich den Rest der Nacht um die Consequenzen der Hypothese auszuarbeiten." - Ob es aber wirklich so gewesen ist, wissen wir nicht. Die visionäre Version stammt erst aus dem Gedächtnisprotokoll einer Rede, die Kekulé 1890, fünfundzwanzig Jahre nach der Publikation der Benzolformel, auf der "Kekulé-Feier“ der Deutschen Chemischen Gesellschaft im großen Saal des Berliner Rathauses gehalten hat, jener pompösen Apotheose der gründerzeitlichen Chemie, die dem sensiblen und zurückhaltenden Kekulé alles andere als willkommen gewesen sein mag. Ob er sich nicht am Ende mit den ngeehrten und gelehrten Herren in Krähwinkel-Berlin", wie er sie kurz darauf in einem Brief an Volhard nannte, einen Scherz erlaubt haben könnte, der an die Idee des pseudonymen F. W. Findig, den Benzolring als Affenreigen darzustellen, anspielte, die vier Jahre zuvor als Sonderheft der ${ }_{n}$ Berichte der Durstigen Chemischen Gesellschaft" den Teilnehmern der Berliner Naturforscherversammlung ausgeteilt worden war? Das zwiespältige Verhältnis Kekulés zur Gründermentalität, dem patriarchalischen Stil der Berliner Gesellschaft, dem Geniekult im allgemeinen und derartigen Jubiläen im besonderen spricht deutlich genug aus seiner Rede. Wenn die Schilderung seiner Vision mit den Worten "Lernen wir träumen, meine Herren!“ schließt, so ist, angesichts der versammelten Prominenz aus preußischer Bürokratie, gründerzeitlicher Industrie und Geheimratsuniversität, die Ironie kaum zu überhören. Es bedürfte deshalb einer sehr viel genaueren historischen Prüfung, um entscheiden zu können, ob die Geschichte vom Genter Kaminfeuer wirklich zum Kontext der Entdeckung der Benzolformel gehört, oder ob sie nicht erst einem sehr viel späteren und ganz anderen Rechtfertigungszusammenhang angehört, zumal Kekulé selbst sich in seiner Erstpublikation der Benzolformel im „Bulletin de la Société Chimique" von 1865 noch der von ihm eingeführten linearen Kettenformel-Schreibweise bediente. Das Beispiel mag zeigen, wieviel quellenkritische Arbeit selbst an einem so bekannten Detail noch zu leisten wäre, ehe sich die Interpretation an eine Frage wie die des archetypischen Nachwirkens des alchemistischen Ouroboros-Symbols wagen dürfte, die so oft mit dieser Episode in Verbindung gebracht wird.

Kritische Haltung gegenüber den Quellen ist selbst dann angezeigt, wenn die berichteten Sachverhalte scheinbar naturwissenschaftlich-exakter Natur sind. Die Invektiven, die Hermann Kolbe aus Leipzig gegen die Typentheorie und gegen die Strukturchemie schleuderte, sind nur zu bekannt. Im Eifer hitziger Schulstreitigkeiten und Prioritätsgefechte dürfte schon manche Entdeckungsgeschichte im nachhinein neu begründet worden sein. Die Geschichte der Naturwissenschaften kennt freilich in vielen Fällen noch eine Form der Sachkritik, die dem Allgemeinhistoriker in der Regel nicht zur Verfügung steht. Sie kann nämlich die Aussagen der Quellen, sofern sie sich auf naturwissenschaftliche Sachverhalte beziehen, mit dem Wissen unserer Zeit überprüfen. So läßt es sich relativ einfach feststellen, daß es sich bei dem 1889 von Bohuslav Brauner entdeckten und in den "Chemical News" publizierten ${ }_{n}$ Austriacum" nicht, wie behauptet, um ein neues Element der Masse 212 gehandelt haben kann. Doch damit postum Lob oder Tadel zu spenden, ist historisch noch nichts gewonnen. Erst wenn wir zu ergründen versuchen, aufgrund welcher Voraussetzungen Deutungen zustandekamen, die sich später als irrig erwiesen, werden wir die Fragestellungen und die Probleme jener Zeit richtig verstehen und ihre Leistungen gerecht beurteilen lernen. Selbst Grenzfälle können in dieser Hinsicht aufschlußreich sein. Im Jahre 1900 veröffentlichte Friedrich Fittica, der verdiente Herausgeber der ${ }_{n} J a h r e s b e r i c h t e$ für die Fortschritte der Chemie", in der "Chemiker-Zeitung" Arbeiten über die chemische Umwandlung von Phosphor in Arsen und Antimon. Natürlich erntete er damit sofort Widerspruch. Und doch zeigt die Tatsache, daß derartige Untersuchungen publiziert und diskutiert werden konnten, wie stark der chemische Elementbegriff in der Zeit um die Jahrhundertwende wieder verunsichert war und welche Faszination noch immer von der alten Proutschen Hypothese vom gemeinsamen Urstoff und der generi schen Verwandtschaft aller Elemente aus ging. Ein eigenartiger historischer Zufall hot es gar gewollt, daß ausgerechnet Otto Habl seine Ausbildung zum Chemiker in ebet demselben Labor erhalten hat, in dem sid zur gleichen Zeit Fittica an der chemische Elementumwandlung versuchte.

Historische Arbeit ist Arbeit mit Quellep Alles, was wir über die Vergangenheit wis sen, wissen wir aus den Zeugnissen, die six uns hinterlassen hat. Nur der kritisch-distart zierte Umgang mit den Quellen aber führ zur historischen Wirklichkeit. Zu leicht näm lich ist man versucht, die Quelle für das ES eignis selbst zu halten, von dem sie Kunde gibt. Deshalb sei zum Schluß noch einm vor fünf methodischen Fehlschlüssen warnt:

- Die Fiktion des Exempels: Historisctr Quellen eignen sich in der Regel nicht dazth bestimmte Positionen der aktuellen natur wissenschaftlichen Forschung zu stützen. und ihr Wert bei der didaktischen Vermitt lung moderner chemischer Konzepte ist strit tig. Denn in beiden Fällen werden heutigt Begriffe und Vorstellungen dem historischet Material aufgezwungen, obgleich dieses $n$ in den Kategorien seiner Zeit, nicht in dened der unseren, richtig zu erfassen ist.

Die Fiktion der Autopsie: Man macht sich zu leicht, wenn man sich darauf verläßt. $\mathrm{da} ß$ Augenzeugen oder unmittelbar Beteiligtt ein Geschehen immer zuverlässig wiederge ben. Jede Zeugenvernehmung vor Gerichl lehrt, daß auch solche Aussagen stets auf ihrt sachliche Verläßlichkeit hin überprüft wer den müssen.

Die Fiktion der Vollständigkeit: Histor sche Uberlieferung ist immer lückenhath Selbst bei gründlichster Nachforschung wer den wir nie alle existierenden Quellen zu $G e$ sicht bekommen, und die uns vorliegender werden nie die ganze Geschichte erzählen.J wir sollten uns sogar darüber Rechenschati geben, weshalb bestimmte Quellengruppet überliefert, andere hingegen nicht erhalter sind.

Die Fiktion der Konsequenz: Geschichtliche Wirklichkeit unterscheidet sich von $\mathrm{ei}^{i}$ nem Groschenroman durch den auffallenden Mangel an Konsequenz im Handeln aller Be teiligten. Der Historiker, der es in der Ge schichte mit realen Menschen zu tun hal. 


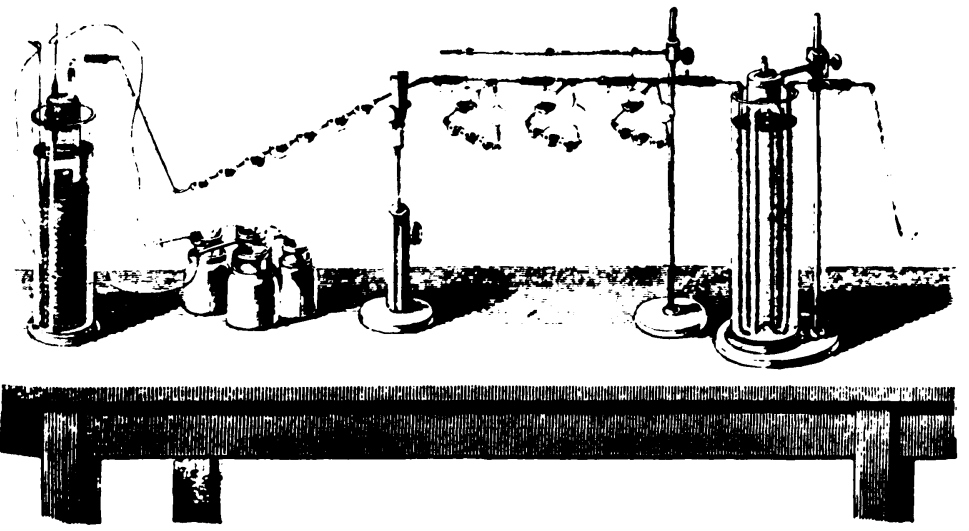

Chemische Apparaturen sind meist den Weg alles Irdischen gegangen. Die wenigen erhaltenen Stuicke sind oft schwer zu identifizieren und zu datieren. Abbildungen in zeitgenössischen Werken leisten dabei gute Dienste. Hier die Versuchsanordnung zur Kolbe-Elektrolyse von aliphatischen Carbonsäuren, die Kolbe selbst allerdings als Methode zur Darstellung des freien „Methyl " gedeutet hat. [Aus: Hermann Kolbe: Ausführliches Lehrbuch der Organischen Chemie. (Graham-Otto's Ausführliches Lehrbuch der Chemie, 3. Aufl., Bd. III) Bd. I. Braunschweig 1854, S. 235]

wird gut daran tun, von diesen kein geringeres $\mathrm{Maß}$ an Widersprüchlichem zu erwarten, als er es von der eigenen Person kennt.

- Die Fiktion der Objektivität: Geschichtsschreibung ist niemals im naturwissenschaftlichen Sinne objektiv. Sie will es auch gar nicht sein, denn der Historiker hat nie eine als Objekt verfügbare äußere Realität vor sich, die sich bestimmen ließe wie die Struktur eines Kristalls. Historische Fragestellungen spiegeln selbstverständlich auch den Standpunkt des Fragenden wider. Damit soll natürlich nicht der Willkür Tür und Tor geöffnet sein. Maßstab und Prüfstein aller historischen Aussagen sind und bleiben die Quellen, die aber stets der Interpretation, der subjektiven Aneignung im Akt historischen Erkennens und Verstehens bedürfen. Wenn zwei Chemiker die physikalischen Daten ein und derselben Verbindung publizieren, sollten im Idealfall exakt identische Ergebnisse herauskommen. Wenn zwei Historiker auch wenn ihnen die gleichen Quellen zur Verfügung stehen - die Geschichte einer Entdeckung oder die Biographie eines Forschers schreiben, so werden im Idealfall durchaus unterschiedliche Darstellungen entstehen.
Wenn ihre Aussagen von den Quellen her fundiert sind, dürfen beide mit gleichem Recht Anspruch auf historische Wahrheit erheben. Je weiter freilich die Forschung fortschreitet, um so leichter wird man sich verständigen können, nach welchen Maßstäben die vorhandenen Quellen in ihrem Verhältnis zueinander zu deuten sind. In der Chemiegeschichtsschreibung stehen wir in vielen Fragen leider noch ganz am Anfang dieses Weges. Geschichte wird nämlich nicht einfach immer wieder umgeschrieben, wie man bisweilen hört, sondern die Entwicklung der historischen Forschung bedeutet insgesamt auch einen Zuwachs an Kenntnis, eine Verfeinerung des Urteils, eine Erweiterung des Gesichtskreises und eine Annäherung an den Eigenwert der Vergangenheit.

\section{Literatur}

Zur Einfübrung in die Geschichte der Naturwissenschaften

George Sarton: Das Studium der Geschichte der Naturwissenschaften. Frankfurt am Main 1965.

Albrecht Timm: Einführung in die Wissenschaftsgeschichte. UTB 203. München 1973.

David Knight: Sources for the History of Science, 1660-1914. (The Sources of History). Cambridge 1975. [Zugleich Quellenkunde und Einführung in die Quellenarbeit!]

Paul T. Durbin (Hrsg.): A Guide to the Culture of Science, Technology, and Medicine. New York, London 1980.

Pietro Corsi und Paul Weindling (Hrsg.): Information Sources in the History of Science and Medicine. (Butterworth Guides to Information Sources.) London, Boston etc. 1983.

\section{Zur Chemiegeschichte und ibrer Methode}

Erich Pietsch: Sinn und Aufgaben der Geschichte der Chemie. Angew. Chem. 50, 939 (1937).

Wolfgang Schneider: Probleme der Chemiegeschichtsforschung. Pharm. Ztg. 107, 1296 (1962).

Chemiegeschichte - Nekrologie oder Wissenschaft? Nachr. Chem. Techn. 15, 317 (1967).

Jost Weyer: Prinzipien und Methoden des Chemiehistorikers. Chem. unserer Zeit 6, 184 (1972).

\section{Chemiehistorisch wichtige Zeitschriften in Auswabl}

AMBIX. The Journal of the Society for the History of Alchemy and Chemistry. $1937 \mathrm{ff}$. [Zur Zeit die einzige ausschließlich der Alchemie- und Chemiegeschichte gewidmete Fachzeitschrift!]

Annals of Science. An International Review of the History of Science and Technology from the Thirteenth Century. $1936 \mathrm{ff}$.

History of Science. $1962 \mathrm{ff}$.

ISIS. An International Review devoted to the History of Science and its Cultural Influences. $1913 \mathrm{ff}$. [Die führende naturwissenschaftshistorische Zeitschrift der Welt]

Dr. Chr. Meinel, geb. 1949 in Dresden, studierte Chemie in Marburg und promovierte 1977 mit einem chemiehistorischen Thema. Er war danach an der University of Kent, den Universitäten Hamburg und Marburg tätig und ist seit 1981 Hochschulassistent am Institut für Geschichte der Naturwissenschaften, Mathematik und Technik der Universität Hamburg. Arbeitstgebiete: Chemische Naturphilosophie des 17. Jahrhunderts, Chemie im 18. und 19. Jahrhundert. 


\section{Chronik}

Das Keto-Enol-Gleichgewicht des Acetons. In jedem Lehrbuch der organischen Chemie liest man, daß Ketone, die am Kohlenstoff in Nachbarschaft zur Carbonylgruppe Wasserstoff tragen, enolisieren können. Ein solches

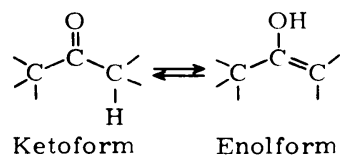

enolisierbares Keton ist auch Aceton (2-Propanon), das einfachste Keton überhaupt. Allerdings liegt z. B. in wäßriger Lösung nur ein winziger Bruchteil der Verbindung in der Enolform (2-Propenol) vor, so daß es große Schwierigkeiten bereitet, den Wert für die Gleichgewichtskonstante $\mathrm{K}_{\mathrm{E}}$ der Enolisierung

$$
\begin{aligned}
& \mathrm{H}_{3} \mathrm{C}^{-} \stackrel{\stackrel{\mathrm{O}}{\mathrm{C}}}{-}-\mathrm{CH}_{3} \stackrel{\mathrm{K}_{\mathrm{E}}}{\rightleftarrows} \mathrm{H}_{3} \mathrm{C}^{-\stackrel{\mathrm{I}}{\mathrm{C}}}{ }_{\mathrm{CH}_{2}} \\
& 2 \text {-Propanon } 2 \text {-Propenol } \\
& \text { (Ketoform) (Enolform) }
\end{aligned}
$$

genau zu messen. Da einfache Enole in der modernen organischen Synthesechemie und auch bei biologischen Reaktionen eine große Rolle spielen, ist man aber an einem möglichst exakten Wert für die Gleichgewichtskonstante und auch an der Säuredissoziationskonstante (dem $\mathrm{pK}_{\mathrm{s}}$-Wert) des Enols sehr interessiert.

Chemikern an der Universität Toronto in Kanada sowie an der Universität Basel [Y. Chiang, A. J. Kresge, Y. S. Tang und J. Wirz, J. Am. Chem. Soc. 106, 460 (1984)] ist es jetzt gelungen, die Keto-Enol-Gleichgewichtskonstante und die Säuredissoziationskonstante für Aceton in wäßriger Lösung mit einer Methode zu bestimmen, bei der es nicht nötig ist, den Zahlenwert einer nicht meßbaren, thermodynamischen oder kinetischen Größe abzuschätzen, wie es bisher immer notwendig war.

Ihr Verfahren beruht auf der Überlegung, daß die Reaktion von einfachen Enolen zu Ketonen durch Basen katalysiert wird und über das sehr reaktive Enolat-Anion verläuft. Bei genügend hoher Basenkonzentration (hier $\mathrm{OH}^{-}$-Ionenkonzentration) liegt das Enol vollkommen in der Enolatform vor, und eine kinetische Analyse in diesem Bereich liefert sowohl die Gleichgewichtskonstante $\mathrm{K}$ als

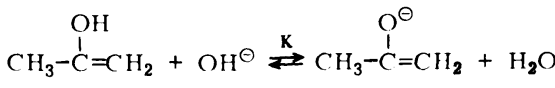

$$
\begin{aligned}
& \text { Fnol Fnolat } \\
& \stackrel{\mathrm{k}_{\mathrm{o}}}{\longrightarrow} \mathrm{CH}_{3}-\stackrel{\mathrm{II}}{\mathrm{C}}-\mathrm{CH}_{3}+\mathrm{OH}^{\ominus} \\
& \text { Keton }
\end{aligned}
$$

Quotient aus der Säuredissoziationskonstante

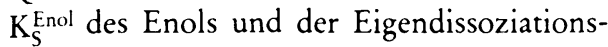
konstante $\mathrm{K}_{\mathrm{W}}$ des Wassers:

$\mathrm{K}=\mathrm{K}_{\mathrm{S}}^{\mathrm{Enol}}: \mathrm{K}_{\mathrm{W}}$

als auch die Geschwindigkeitskonstante $k_{0}$. Der Trick des Verfahrens ist die Erzeugung des Propenols aus geeigneten Vorläufern mit Hilfe einer photochemischen Reaktion. Es ist bekannt, daß Ketone, die in $\boldsymbol{\gamma}$-Stellung Wasserstoff tragen, durch Bestrahlen mit UVLicht in Enol und Olefin gespalten werden. Um auf diesem Weg Propenol zu erzeugen, wurde z. B. Methylisobutylketon mit einem Laserblitz (20 ns, Wellenlänge $265 \mathrm{~nm}$ ) bestrahlt. Das dabei entstehende Propenol hat

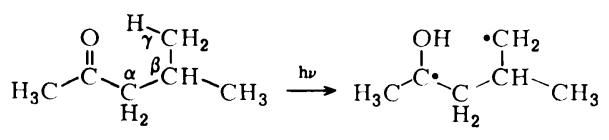

Methylisobutylketon

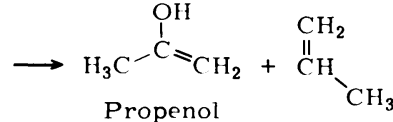

$$
\begin{aligned}
& \text { Propenol } \\
& \downarrow
\end{aligned}
$$

in 0,1 molarer $\mathrm{NaOH}$ eine Lebensdauer von 20 Mikrosekunden und zerfällt nach einer Reaktion 1. Ordnung. Aus der Geschwindigkeitsgleichung für seine Umwandlung ins Keton erhält man die Säuredissoziationskonstante des Enols $K_{S}^{\text {Enol }}=(1,09 \pm 0,07) \cdot 10^{-11}$ mol (das entspricht dem $\mathrm{pK}_{\mathrm{S}}^{\mathrm{Enol}}=10,96 \pm$ 0,03 ) und die Geschwindigkeitskonstante $\mathrm{K}_{0}^{\prime}=$ $(5,01 \pm 0,17) \cdot 10^{4} s^{-1} \cdot \mathrm{pK}_{\mathrm{S}}^{\text {Enol }}$ konnte auch noch auf ganz unabhängigem Weg aus der Anfangsabsorption nach der oben erwähnten Belichtung $\mathrm{zu} \mathrm{pK}_{\mathrm{S}}^{\mathrm{Enol}}=10,83 \pm 0,06$ bestimmt werden. Aus dem gewichteten Mittel der beiden

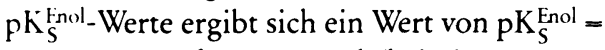
$10,9+ \pm 0,08$ für Propenol (bei einer Ionenstärke von $0,10 \mathrm{~mol}$ )

Außerdem haben die Autoren die Geschwindigkeit der durch $\mathrm{OH}^{-}$-Ionen katalysierten Enolisierung von Aceton (bei einer Ionenstärke von 0,10 mol) gemessen und auf diese Weise für die Säuredissoziation der Ketoform des Acetons $\mathrm{pK}_{\mathrm{S}}^{\mathrm{keton}}=19,26 \pm 0,04$ bestimmt. Aus $\mathrm{K}_{\mathrm{S}}^{\text {Enol }}$ und $\mathrm{K}_{\mathrm{S}}^{\text {heton }}$ erhält man dann die Gleichgewichtskonstante für die Keto-EnolTautomerisierung des Acetons: $\mathrm{K}_{\mathrm{E}}=(6,0 \pm$ $1,1) \cdot 10^{-9}$ (entsprechend $\mathrm{pK}_{\mathrm{E}}=8,22 \pm 0,08$ ) bei $25^{\circ} \mathrm{C}$ in wäßriger Lösung.

Mit Hilfe der klassischen Titrationsmethode mit Brom zur Messung des Enolgehalts (Brom reagiert bei tiefer Temperatur schneller mit der Enolform als diese aus der Ketoform nachge liefert wird) konnte man nur feststellen, dal $\mathrm{pK}_{\mathrm{E}}>6$ ist. Mit anderen, neueren Meßme thoden war $\mathrm{pK}_{\mathrm{E}}$ zu 7,2 $\pm 0,9$ und 8,5 $\pm 0,3$ bestimmt worden.

Eine rein organische Verbindung, die sich wie ein anorganisches Salz gebärdet, nämlich in Ionen dissoziiert, fanden E. M. Arnett und seine Mitarbeiter. In unpolaren Lösungsmit teln schwer löslich (und daher daraus in kri stalliner Form gewinnbar), löst sich die Ver bindung in Aceton oder stärker polaren Lösungsmitteln, wobei eine Kohlenstoff-Koh lenstoff-Bindung gebrochen und ein Carbo kation und ein Carbanion gebildet werden.

Schon in einer früheren Publikation [Tetrahe dron Lett. 24, 3299 (1983)] hatten E. M. Arnetl und E. B. Troughton darüber berichtet, da sich durch Kombination von Carbokationen $\mathrm{R}_{3} \mathrm{C}^{+}$, und Carbanionen, $\mathrm{R}_{3}^{\prime} \mathrm{C}^{-}$, CC-Bindun gen knüpfen ließen; je nach Stabilität vo̊ Kation und Anion sowie je nach Lösungsmil tel lagen in Lösung bevorzugt die Ionen oder das kovalente Reaktionsprodukt vor, oder man konnte ein Gleichgewicht nachweisen. Wenig später berichteten die Autoren [J. Am. Chem. Soc. 105, 6172 (1983)] über ein beson" ders geeignetes System, um die Kinetik und Thermodynamik der Bindungsbildung bzw. Dissoziation studieren zu können: Trimethyl cyclopropeniumtetrafluoroborat und Kalium paranitrophenylmalonitril, beide gelöst in Aceton, werden zusammengegeben, das aus gefallene Kaliumtetrafluoroborat abfiltriert, das Lösungsmittel abgezogen, das farblose Produkt in Benzol/Hexan aufgenommen und durch Abkühlen wieder kristallisiert. Eine Röntgenstrukturanalyse ergab eindeutig, daß sich das kovalent gebundene Produkt Trime thylcyclopropenyl(p-nitrophenyl)malonitril (,TCNM“) gebildet hatte.

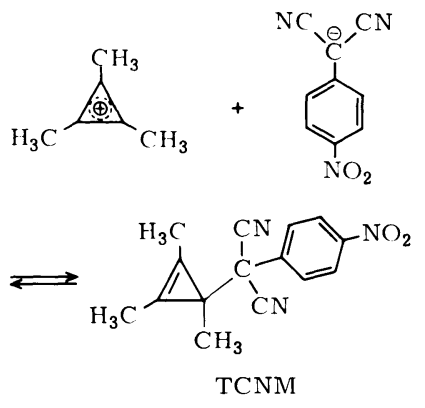

Löst man das farblose TCNM in Aceton, ${ }^{\text {st }}$ erscheint die orange Farbe des Carbanions; dir 
Bildungsreaktion von TCNM ist also, abhängig von der Polarität des Lösungsmittels, reversibel. Auf die Labilität der neu gebildeten CC-Bindung deutet auch deren ungewöhnlich große Bindungslänge von 158,8 pm hin.

Das Gleichgewicht zwischen kovalentem Produkt und den Ionen wurde an der Verbindung, die am Cyclopropan-Ring Phenyl- statt Methylgruppen trägt, näher untersucht. Danach liegt die Bildungsenthalpie für die CC-Bindung in der Größenordnung 5 bis $6 \mathrm{kcal}$ $\mathrm{mol}^{-1}$.

Ein weiteres Ziel der Untersuchungen ist es, die Heterolyse von TCNM in der Gasphase zu untersuchen, in der keine Lösungsmittelmoleküle vorliegen, welche die Ionen stabilisieren könnten [Chem. Eng. News, 5. März 1984, S. 29].

Die Hutfarbstoffe des Maronenröhrlings, Xerocomus badius (Fr.) Kühn. ex Gilb., eines beliebten Speisepilzes mit einer schokoladenbraunen Huthaut, haben B. Steffan und W. Steglich isoliert und ihre Strukturen aufgeklärt [Angew. Chem., im Druck]. Die Farbstoffe, die in der Huthaut offensichtlich als Salze vorliegen, lassen sich mit einem Gemisch aus Methanol und Aceton unter Zusatz von etwas Salzsäure extrahieren und durch mehrfache Chromatographie an Sephadex mit Methanol als Laufmittel trennen und reinigen. Als Hautkomponenten des Farbstoffgemisches wurden Badion A und Norbadion $\mathrm{A}$ identifiziert und daneben geringere Mengen an Bisnorbadiochinon $\mathrm{A}$ und $\mathrm{O}-\mathrm{Me}-$ thylpulvichinon, wobei das letztere seine $\mathrm{Me}$ thylgruppe wahrscheinlich erst während der Extraktion mit angesäuertem Methanol erhält.

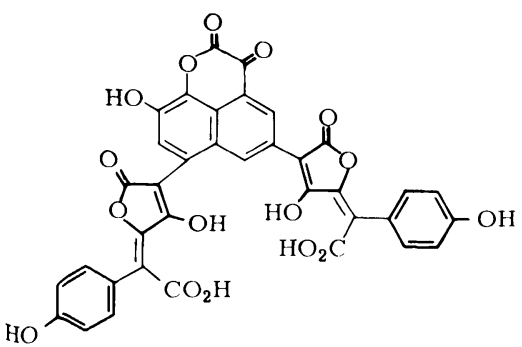

Badion A
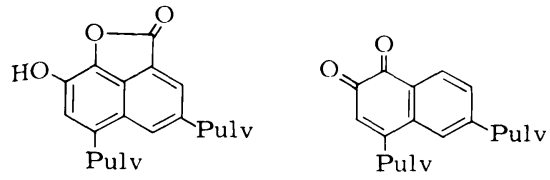

Norbadion

Bisnorbadiochinon A

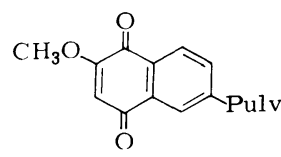

O-Methyl -

pulvichinon $\mathrm{A}$

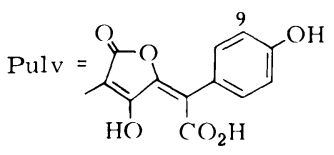

Es konnte auch nachgewiesen werden, daß Xerocomsäure der biogenetische Vorläufer von Badion A ist: Dazu wurde ein Stück Huthaut von einem Maronenröhrling entfernt und das Fleisch darunter mit einer wäßrigen Xerocomsäure-Lösung behandelt. Der gelbe Fleck färbte sich in wenigen Minuten blau und dann allmählich braun. Der Farbstoff konnte extrahiert und im Extrakt Badion A nachgewiesen werden.

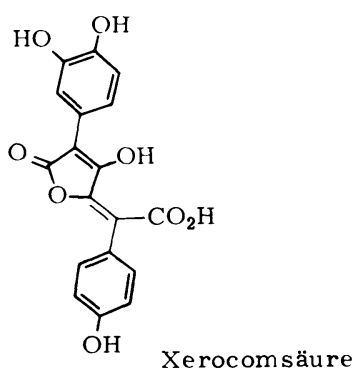

Badion A ist auch der Hutfarbstoff des Kiefernsteinpilzes Boletus pinicola Vitt. Im Flokkenstieligen Hexenröhrling, Boletus erythropus (Fr. ex Fr.) Pers., dagegen kommt stattdessen Badion B vor, das an den beiden Pulvinsäure-Seitenketten jeweils eine zusätzliche $\mathrm{OH}-\mathrm{Gruppe}$ in 9-Stellung trägt.

Übrigens wurde in der gleichen Arbeitsgruppe [B. Fugmann und W. Steglich, Angew. Chem. 96, 71 (1984)] vor kurzem der Weiße Rasling, Lyophyllum connatum (Schum. ex Fr.) Sing., der als guter und ergiebiger Speisepilz gilt, untersucht. Dabei wurden die drei Inhaltsstoffe N'-Hydroxy-N,N-dimethylharnstoff 1, Connatin 2 und das Dimethyl-methylazoxycarboxamid 3 isoliert und identifiziert.

$\left(\mathrm{CH}_{3}\right)_{2} \mathrm{~N}-\stackrel{\mathrm{O}}{\mathrm{C}}-\mathrm{NHOH}$

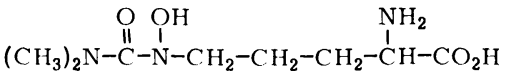
2

$\left(\mathrm{C} \cdot \mathrm{H}_{3}\right)_{2} \mathrm{~N}-\stackrel{\mathrm{O}}{\mathrm{C}}-\mathrm{N}=\stackrel{\oplus}{\mathrm{N}}{ }_{3}^{\prime} \mathrm{OH}_{3}$

Da einfache aliphatische Azoxyverbindungen stark mutagen wirken und auch N-Hydroxy- harnstoffe in den Stoffwechsel der DNA eingreifen, warnen die Autoren vor dem Genuß des Weißen Raslings. Immerhin enthält $1 \mathrm{~kg}$ des Pilzes $400 \mathrm{mg} 3$ und mehr als $2 \mathrm{~g} 2$.

Element 108 entdeckt. Zum dritten Mal nacheinander ist es den Darmstädter Physikern der Arbeitsgruppe von Peter Armbruster bei der Gesellschaft für Schwerionenforschung (GSI) gelungen, ein neues Element zu erzeugen. Nach Element 107 im Jahre 1981 und Element 109 im Jahre 1982 ist jetzt das Element 108 synthetisiert worden. Die Physiker Gottfried Münzenberg, Sigurd Hofmann, Willibrord Reisdorf und Karl-Heinz Schmidt fanden in einem zur Zeit noch laufenden Experiment bisher drei Atome, die eindeutig dem neuen Element 108 zugeordnet werden müssen. Das neue Element wurde durch Verschmelzung eines Eisen-58-Kerns mit einem Blei-208-Kern erzeugt, hat das Atomgewicht 265 und zerfällt in weniger als zweitausendstel Sekunden durch Aussendung eines $\boldsymbol{\alpha}$-Teilchens von $10,4 \mathrm{MeV}$ in ein Isotop des Elements 106 mit dem Atomgewicht 261, das wiederum durch Aussendung weiterer $\alpha$-Teilchen in bekannte Isotope der Elemente 104 und 102 zerfällt. Diese Tochterzerfälle wurden nachgewiesen.

Damit sich Atomkerne berühren und eventuell verschmelzen können, müssen sie zunächst die gegenseitige Abstoßung überwinden, die von ihren positiven elektrischen Ladungen herrührt. Hierzu muß ein Atomkern auf ca. zehn Prozent der Lichtgeschwindigkeit beschleunigt werden. Im Experiment geschah dies durch den $120 \mathrm{~m}$ langen UnilacBeschleuniger der GSI, der die Kerne des Eisens (Ordnungszahl 26) mit der nötigen Geschwindigkeit auf eine dünne Folie aus Blei (Ordnungszahl 82) schoß. Die Kerne des dabei entstehenden Elementes 108 sind so empfindlich, daß die meisten auch bei einem sanften Kontakt sofort wieder in zwei Teile auseinander brechen. Daher können im Versuch mit ca. $10^{18}$ Geschoßteilchen nur wenige Atome des Elementes 108 erzeugt werden. Mit einem speziellen, $11 \mathrm{~m}$ langen Geschwindigkeitsfilter aus elektrischen und magnetischen Feldern werden diese dann von den Geschoßteilchen abgetrennt und auf ein Nachweisgerät gelenkt. Dies identifiziert das neue Element anhand der von ihm ausgehenden $\alpha$-Strahlung. Sie ist charakteristisch wie ein Fingerabdruck und erlaubt es, das neue Element zweifelsfrei nachzuweisen. Überraschend ist, daß das neueElement nicht, wie von der Theorie vorhergesagt, durch spontane Spaltung zerfällt. 